\title{
MANIKIN BASED STUDIES OF SIMULATED RESUSCITATION PRACTICES: TERM FACE
} MASK STUDY

\author{
F.E. Wood ${ }^{1}$, C.R. Platten ${ }^{2}$, S. Byrne ${ }^{1}$, J.P. Wyllie ${ }^{1}$ \\ ${ }^{1}$ Department of Neonatal Medicine, ${ }^{2}$ Regional Medical Physics Department, South Tees Hospitals NHS \\ Foundation Trust, James Cook University Hospital, Middlesbrough, UK
}

Background and aims: In studies of mask effectiveness leak has been the primary comparator and no studies have investigated the force required to create an effective mask seal. We aimed to determine whether leak at the face mask and force applied to achieve mask seal differs between masks designed for use in term infants when using either a single handed (SH) or a two handed mask hold (TH).

Methods: Seventy-two participants gave positive pressure ventilation using a Neopuff, to a modified manikin in a system designed to measure mask leak and applied force. Four masks were compared by analysing data from a Florian monitor and a concealed force plate with $\operatorname{Spectra}^{\circledR}$ software.

Results: Mask leak varied with a significant difference between masks for both SH and TH techniques. Leak was significantly reduced with the TH technique and the disparity between professional categories $(\mathrm{p}<$ 0.001) seen with the SH hold was resolved.

Mean applied force was significantly different between masks with SH but not TH. With both techniques mean applied force differed between professional categories $(\mathrm{p}<0.001)$. No meaningful relationship between applied force and mask leak was identified.

The mean ranks of percentage of successful inflations for each mask were statistically different $(p<0.001)$ but median values were similar.

Conclusion: None of the masks tested exhibited both the lowest leak and lowest applied force but one mask performed least well. Applying a TH technique produced less mask leak and less variability between professional categories but increased the mean applied force. 